\title{
Design of a SiC-Based Switched CCM/TCM Inverter for High-speed Machine Drive with Low PWM-Induced Current Ripple
}

\author{
Yunlei Jiang, Yanfeng Shen, Luke Shillaber, Teng Long \\ Department of Engineering, University of Cambridge, Cambridge CB3 0FA, United Kingdom \\ Email: yj306@cam.ac.uk,ys523@cam.ac.uk, 1s669@cam.ac.uk, t1322@cam.ac.uk
}

\begin{abstract}
This paper presents the design of a SiC-based inverter for high-speed machine using a continuous conduction mode (CCM) and triangular conduction mode (TCM) switched switching scheme. The implementation of the switched TCM and CCM on machine drive are explored analytically in the context of PWM-induced current quality and efficiency, based on which, an improved switched switching strategy is employed. According to the difference in uneven current ripple distribution of TCM and CCM, the proposed strategy alternates between TCM and CCM in one line cycle for an enhanced current ripple and efficiency performance.
\end{abstract}

Keywords-High-speed machine, Triangular conduction mode, Continuous conduction mode, Switched switching strategy

\section{INTRODUCTION}

Due to advantages in high power density and efficiency, the high-speed electric machine (HSEM) has gained lots of interest in recent years. For high-speed machines, the high mechanical speed is usually required to obtain high power density because the increment of torque density is relatively difficult. Although a low pole pair number is preferable for high-speed machines [1], its popularity is limited by factors including axial length and unbalance flux linkage [2], which makes high pole machines an inevitable choice. In this regard, output frequency up to $1000 \mathrm{~Hz}$ [3] is typical for common high-speed applications. Meanwhile, the stator inductance of a high-speed machine is relatively small due to fewer turn numbers [3], which impairs its functionality as a passive filter and a higher switching frequency is hence required for a low PWM-induced current ripple. The switching frequency capability of traditional Si-based semiconductor devices for the motor drive is commonly limited to $20 \mathrm{kHz}$ and the aforementioned features of HSEM indicate that the inverter is unable to provide enough pulse per cycle, which is likely to give rise to an unreliable current regulation performance, a significant amount of current harmonics and additional PWMinduced loss in the machine consequently [4].

An intuitive solution to the aforementioned issues is to replace the Si-based device by their $\mathrm{SiC}$ counterparts employing an increased switching frequency or applying a bulky output filter [5]. The resulted efficiency penalty and increase of volume and weight, however, could be unacceptable. The TCM switching strategy, which features triangular current waveform and zero voltage switching (ZVS) for greatly reduced switching losses [6], has been explored widely for PV inverter [7] and power factor correction (PFC) [8] applications while its advantages in variable frequency machine drive are seldom reported. In [9, 10], TCM inverter for machine drive purpose is demonstrated and three-order harmonic current injection methods are discussed to improve modulation index. In [11], a 2-kW discontinuous conduction mode (DCM), which is similar to the TCM strategy in terms of soft-switching capability, inverter is reported for the PMSM. In these pre-art literature, the main disadvantage of the DCM or TCM switching strategy is an increased conduction current to meet the soft-switching condition, bringing a trade-off of increased conduction loss, therefore it is conventionally considered as unsuitable for high-power applications.

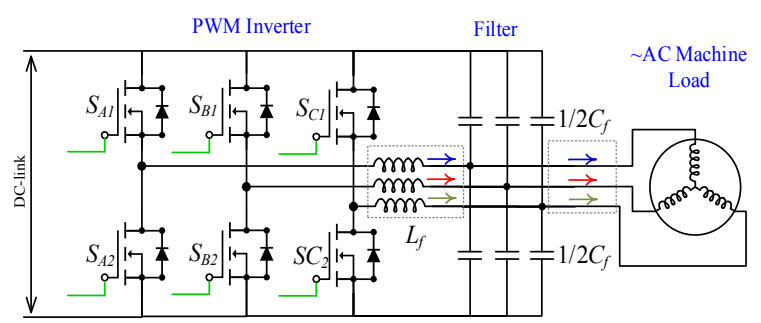

Figure 1. Complete structure of the investigated inverter.

In this paper, a switched TCM/CCM scheme is investigated for machine drive applications, taking advantage of both switching strategies in a dedicated topology, while also mitigating their respective challenges. A similar strategy employing a hybrid switching pattern was previously reported in [12] and [13]. In this paper, uneven current ripple distribution in time domain and system behavior is firstly derived. Then, a switched strategy is proposed and simulations are presented to verify the proposed switching strategy. It is found that the inverter performance is improved in terms of current THD and efficiency at certain operating points.

\section{INVERTER ARCHITECTURE AND PROPOSED SWITCHED STRATEGY}

\section{A. Inverter Architecture and Design Requirements}

Fig. 1 shows the configuration of the inverter setup, which has been previously demonstrated in [10]. The $\mathrm{SiC}$ inverter consists of three individual half-bridges, each connected to a common DC-link voltage, three half-bridge switches, three filter inductors, denoted as $L_{f}$, and six filter capacitors on the inverter side. The inverter resembles a conventional two-level 
inverter with output filters. Note that machine-side inductor $L_{s}$ is the self-inductance of the machine, normally around several hundred $\mu H$ depending on the structure of the machine [3]. For the following analysis, inverter and machine specifications, based on which case study is constructed, are given in Table I and II, respectively. It should be underlined that the configuration provides low impedance paths for neutral currents, so the control of a single phase is independent of the other phases.

\section{B. Operation Principle of the Switched Strategy}

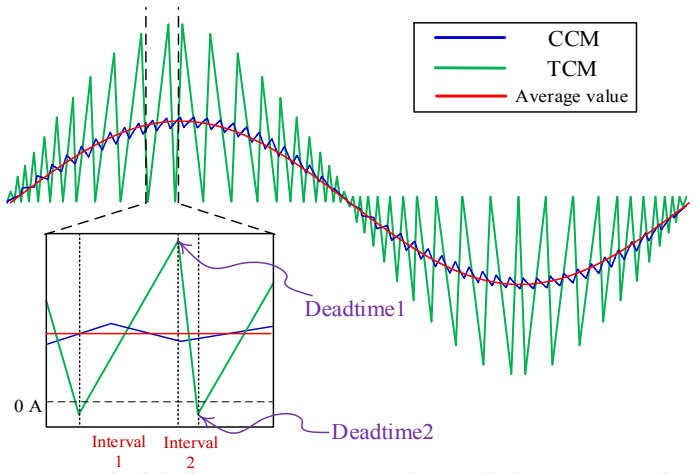

Figure 2. Typical inductor current waveforms during TCM and CCM operations.

Fig. 2 shows the typical current and voltage waveforms for the two operation modes of the investigated inverter under unitary fundamental displacement factor. In order to obtain identical average value during one switching cycle, TCM features a larger inductor current fluctuation, which consists of a sinusoidal fundamental and a high-frequency component, enabling zero voltage switching (ZVS) at the bottom of the current waveform.

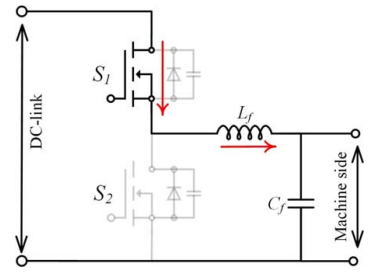

(a)

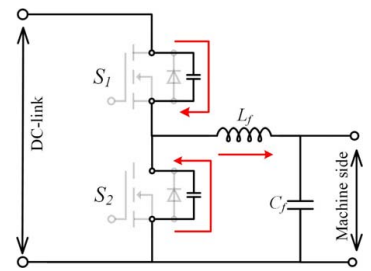

(c)

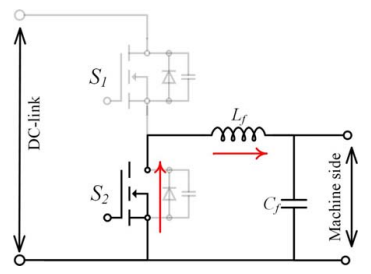

(b)

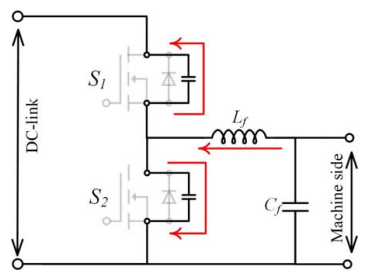

(d)
Figure 3 Current paths for TCM operation with negative output current for ZVS turn-on. (a) Interval 1: Charing of $L_{f}$ by turning on $S_{1}$. (b) Interval 2: Discharging of $L_{f}$ by turning on $S_{2}$. (c) Deadtime1: Discharging of $S_{1}$ output capacitance and charging of $S_{2}$ output capacitance. (d) Deadtime2: Charging of $S_{1}$ output capacitance and discharging of $S_{2}$ output capacitance. ZVS turn on happens at the end of this subinterval.
There exist 4 intervals in one switching cycle for TCM. In interval 1, $S_{1}$ is turned on, as shown in Fig. 3(a), so the output inductor is charging. In interval $2, S_{1}$ is turned off and $S_{2}$ is turned on, which discharges $L_{f}$. The ZVS turn-on is expected to happen at the bottom of the current waveform as shown in Fig. 3 (d), where the output capacitance of upper device $S_{1}$ and lower device $S_{2}$ is discharged to zero and charged to positive rail respectively in the deadtime.

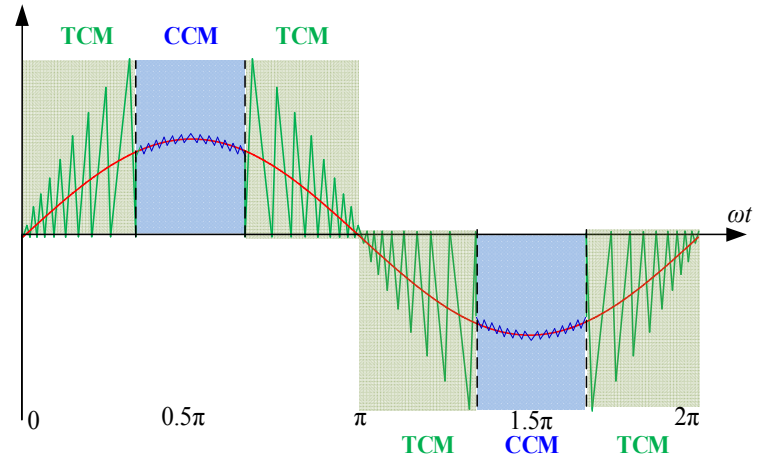

Figure 4. Illustration of the proposed switched TCM/CCM strategy during high modulation operation.

In TCM operation, each phase leg is turned on with zero voltage switching so the turn-on loss could be regarded as zero. The conduction loss forms the majority of the loss, as the turn-off loss is usually small for SiC MOSFET. In CCM operation, all the MOSFETs are hard switched on. Hence, both conduction loss and switching loss exist and the switching loss accounts for a significant part.

The limitation on Total Harmonic Distortion (THD) of the fundamental current, which is identified as the main source of PWM-induced issues, is considered as the main requirement of system design for high-speed machines. The derivation of THD distribution over one line cycle will be discussed in the following subsection. Aiming at a low PWM-induced current ripple, a switched TCM/CCM strategy is proposed as illustrated in Fig. 4. This strategy results from the fact that the filter network of a TCM inverter has the potential to work as an $L C L$ filter, in which the inverter-side filter is formed by $L_{f}$ and $C_{f}$ while the load-side inductor is provided by the armature inductor of the machine when operating at CCM. During operation, the TCM and CCM are interchanged in one line cycle depending on the result of current ripple prediction and which one owns a superior performance in terms of current THD and system efficiency.

TABLE I SPECIFICATIONS OF THE MACHINE UNDER INVESTIGATION

\begin{tabular}{ccc}
\hline \hline Machine Parameters & Symbol & Value \\
\hline Machine type & $/$ & PMSM \\
Rated phase voltage & $\mathrm{V}_{\mathrm{m}}$ & $110 \mathrm{~V}$ \\
(RMS) & $i_{\text {phase }}$ & $20 \mathrm{~A}$ \\
Rated phase current (RMS) & $f_{L}$ & $500 \mathrm{~Hz}$ \\
Rated frequency & $L_{s}$ & $200 \mu \mathrm{H}$ \\
Machine-side inductor &
\end{tabular}


TABLE II

SPECIFICATIONS OF THE INVERTER UNDER INVESTIGATION

\begin{tabular}{ccc}
\hline \hline Inverter Parameters & Symbol & Value \\
\hline DC-link voltage & $\mathrm{V}_{\mathrm{dc}}$ & $400 \mathrm{~V}$ \\
DC-link capacitor & $\mathrm{C}_{\mathrm{dc}}$ & $50 \mu \mathrm{F}$ \\
$\mathrm{SiC}$ - MOSFET & $/$ & $\mathrm{C} 3 \mathrm{M} 0060065 \mathrm{~K}$ \\
Filter capacitor & $C_{f}$ & $10 \mu \mathrm{F}$ \\
Inverter-side inductor & $L_{f}$ & $75 \mu \mathrm{H}$ \\
\hline \hline
\end{tabular}

C. PWM-induced Current Ripple and System Behavior of $T C M \& C C M$ Operations

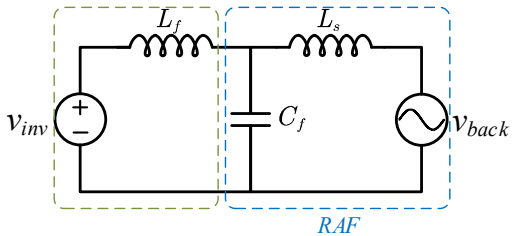

(a)

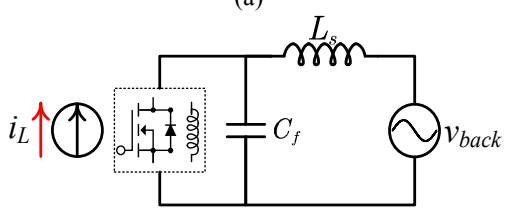

(b)

Figure 5. Output filter during (a) CCM operation; (b) TCM operation.

In order to predict the current ripple in TCM and CCM operations, the equivalent circuit models of the inverter in CCM and TCM operations are firstly derived in Fig. 5. Due to the difference in the modulation technique, the plant models are different for two cases.

As for the CCM operation, the system can be modeled as a voltage source inverter (VSI) with an output filter network. The filter network is then decomposed into two stages, as depicted in Fig. 5(a). It is assumed that sinusoidal pulse width modulation (SPWM) is used for the switching algorithm to achieve identical usage of dc-link voltage. In this operation mode, one switching operation is divided into two subintervals ( $T_{\text {on }}$ and $T_{\text {off }}$ ), during which the magnitude of inductor current increases during $T_{\text {on }}$ and then decreases during $T_{\text {off }}$. Based on the assumption that the switching frequency is higher much higher (30 times) than the fundamental frequency so the capacitor voltage is constant within one switching cycle and the volt-second on inductor $L f$ is roughly balanced, the peak-to-peak inverter-side current ripple can be expressed as:

$$
\begin{gathered}
\Delta I_{p k-p k}=\frac{\left[V_{d c}-d(t) V_{d c}\right] d(t) T_{s}}{2 L_{f}} \\
=\frac{V_{d c}}{2 L_{f} f_{s w}}[1-d(t)] d(t)
\end{gathered}
$$

where $f_{s w}$ is the switching frequency and $d(\mathrm{t})$ is the duty ratio in a certain switching cycle. Note that the machine-side voltage appears as a short circuit at the harmonic frequency due to assuming pure sinusoidal grid voltage at the fundamental frequency. If rewritten in the form of modulation index, equation (2) can be obtained as:

$$
\Delta I_{p k-p k}=\frac{V_{d c}}{2 L f_{s w}}\left(1-m_{a}\left|\sin 2 \pi f_{L} t\right|\right) m_{a} \mid \sin 2 \pi f_{L} t
$$

where $m_{a}$ is the amplitude of the time-varying modulation index and $f_{L}$ denotes the line frequency. In the second stage, filter capacitor $C_{f}$ and machine-side inductor provide a constant ripple attenuation factor (RAF) since the switching frequency is fixed [14]:

$$
H_{R A F}(j w)=\frac{1}{\omega_{s w}^{2} L_{s} C_{f}-1}
$$

Based on (3), Fig. 5(a) depicts the distribution of $\Delta I_{p k-p k}$ over a half fundamental cycle versus modulation index when the switching frequency is fixed at $15 \mathrm{kHz}$, a typical value for AC machine drive. As observed, the current ripple is not evenly distributed in one half line cycle but varies based on the modulation index. It can also be observed that the harmonic attenuation performance is highly dependent on the value of the modulation index. Additionally, the magnitude of $\Delta I_{p k-p k}$ reaches its peak at around $\omega t=0.5$ at high modulation index.

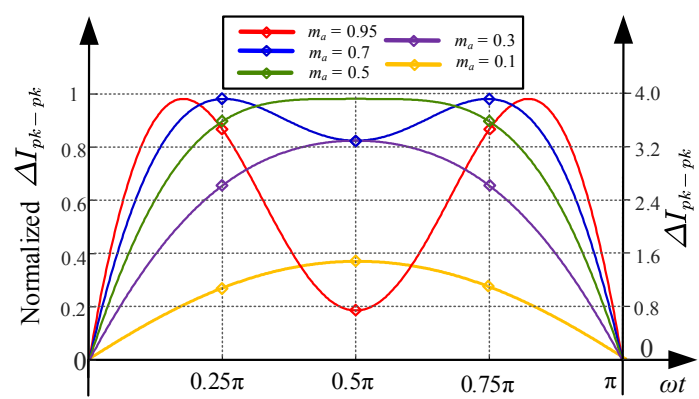

(a)

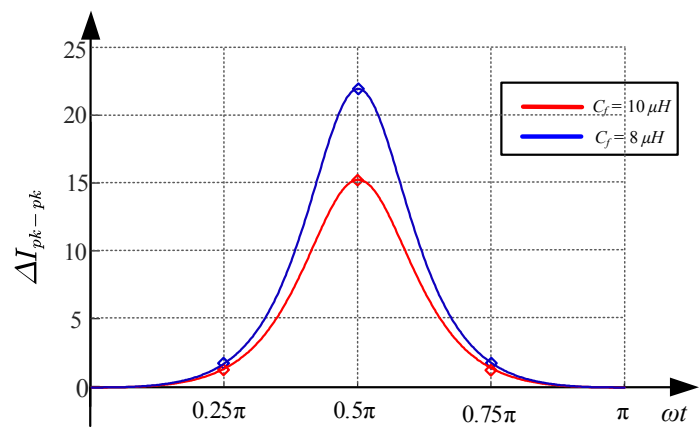

(b)

Figure 5. Current ripple distribution of (a) CCM @ $f_{s w}=15 \mathrm{kHz}$ and (b) TCM over half one line cycle.

In TCM operation, the inverter works as a controlled current source since the shape of the leg output current is intentionally controlled to achieve ZVS and a $C L$ filter could be seen as a low-pass filter. The transfer function from inductor current to machine-side current can be derived as: $i_{s} / i_{L}=1 /\left(L_{s} C_{f} s^{2}+1\right)[15]$ and the cut-off frequency of the filter is obtained correspondingly as: $f_{c}=1 / 2 \pi \sqrt{L_{s} C_{f}}$, which provides an attenuation of $-40 \mathrm{~dB} / \mathrm{dec}$ above the cutoff frequency. As stated above, a variable switching frequency is 
the nature of TCM strategy, the ON-time and OFF-time within one switching frequency in this mode are expressed as:

$$
\left\{\begin{array}{c}
t_{o n}=\frac{L_{f}\left(2 i_{L}^{*}+2 i_{\text {lower }}\right)}{V_{d c} / 2-v_{a c}} \\
t_{\text {off }}=\frac{L_{f}\left(2 i_{L}^{*}+2 i_{\text {lower }}\right)}{V_{d c} / 2+v_{a c}}
\end{array}\right.
$$

where $i_{\text {lower }}$ denotes the current lower limit which is essential for ZVS turn-on. Moreover, the variable switching frequency is given as:

$$
f_{s w}=\frac{1}{t_{o n}+t_{o f f}}=\frac{V_{d c}{ }^{2} / 4-v_{a c}}{L_{f}\left(2 i_{L}^{*}+2 i_{\text {lower }}\right) V_{d c}}
$$

Assuming the value $C_{f}$ is $8 \mu F$ and the corresponding cutoff frequency is around $4.1 \mathrm{kHz}$, the current ripple distribution of the TCM can be obtained as shown in Fig. 5(b) by calculating the gain of current ripple with various switching frequency, i.e. (5), over the $C L$ filter. The maximum current ripple occurs when $\omega t=0.5 \pi$ since the variable switching frequency of TCM reaches its lowest value at this point, which indicates the weakest attenuation capability but the highest current ripple. Besides, larger $C_{f}$ reduces the current ripple magnitude, although the power density of the inverter is sacrificed. It is worth noting that the current ripple magnitude of the CCM is significantly smaller than that of the TCM when $\omega t$ is around $0.5 \pi$ during high modulation index $\left(m_{a}=0.95\right)$ operation. Considering that the conduction loss of CCM is also smaller than that of TCM, this feature justifies the benefits of alternating the TCM and CCM within one line cycle as illustrated in Fig. 3.

\section{EVAluAtion OF THE Switched TCM/CCM Switching TECHNIQUE}

To evaluate the operation point, closed-form expressions of switching and conduction loss for both modulation techniques ought to be determined. The calculation is conducted based on the static characteristics of C3M0060065K from Cree.

\section{A. Conduction loss and switching loss of TCM operation:}

The root-mean-square (RMS) of the TCM current within one switching cycle can be described as:

$$
i_{L_{-} \mathrm{RMS} \_\mathrm{TCM}}=\sqrt{i_{L}^{* 2}+\frac{1}{12}\left(2 i_{L}^{*}+2 B_{0}\right)^{2}}
$$

where $i_{L}^{*}$ is the current reference within one switching cycle and $B_{0}$ is the lower limit of the current reference, which is also referred to as the ZVS boundary current. Furthermore, conduction loss $P_{\text {con_TCM }}$ in one phase leg is approximated by integrating the time-varying loss:

$$
P_{\text {con_TCM }}=f_{L} \int_{0}^{1 / f_{L}} R_{d s, o n}\left(i_{L}^{* 2}+\frac{1}{12}\left(2 i_{L}^{*}+2 B_{0}\right)^{2}\right) d t
$$

where $R_{d s, o n} \approx 60 \mathrm{~m} \Omega$ is the on-state resistance (at $100^{\circ} \mathrm{C}$ junction temperature) of the device. It should be underlined that $P_{\text {con_TCM }}$ is independent of the value of the filter network.

The switching loss can be classified into overlapping loss $E_{V-I}$ and junction capacitance loss $E_{o s s} \& E_{\text {qoss }}$ as pointed out in [16]. The TCM is operated with ZVS turn-on with almost zero turn-on loss, so $E_{V-I, O N}, E_{o s s,}$ and $E_{\text {qoss }}$ are eliminated. The turn-off transient generates a loss $E_{V-I, O F F}$, although relatively small, due to the inevitable overlap between drain to source voltage and current at the time of turn-off instant. Note that the deadtime optimization is not part of this work and the loss analysis does not include reverse conduction losses at various operating points. In this regard, the switching loss in TCM operation is approximated by $P_{\text {switching } \_ \text {TCM }}=f_{L} E_{V-I, O F F}$.

\section{B. Conduction loss and switching loss of CCM operation:}

The calculation of the conduction loss of CCM operation follows the result provided in (1),

$$
i_{L \_ \text {RMS_TCM }}=\sqrt{i_{L}^{* 2}+\frac{1}{12}\left(\triangle I_{p k-p k}\right)^{2}}
$$

and then by integrating the current flowing through $L_{f}$ over one switching cycle as given in (9)

$$
\begin{aligned}
& P_{c o n \_\mathrm{CCM}}=f_{L} \int_{0}^{1 / f_{L}} R_{d s, o n}\left(i_{L}^{*^{2}}+\frac{1}{12}\left(\triangle I_{p k-p k}\right)^{2}\right) d t \\
= & f_{L} \int_{0}^{1 / f_{L}} R_{d s, o n}\left[i_{L}^{* 2}+\frac{1}{12}\left(\frac{V_{d c}}{2 L f_{s w}}(1-d(t)) d(t)\right)^{2}\right] d t
\end{aligned}
$$

Similarly, the switching losses in a phase leg can be approximated by integrating the switching energy, which is described as a function of drain-source current $I_{d s}$ given by the datasheet of C3M0060065K. The overlapping loss $E_{V-I, O N}$, and $E_{V-I, O F F}$ increases linearly with the drain-source current while the junction capacitance loss $E_{\text {oss }}$ and $E_{\text {qoss }}$ are constant and unaffected by $i_{d s}$. Based on this characteristic, both turnon and turn-off loss can be estimated analytically.

Based on the analysis given above, loss distribution, including conduction loss and switching loss, and difference between TCM and CCM operations at rated working point $\left(I_{R M S}=20 \mathrm{~A}\right)$ over half a line cycle are illustrated in Fig. 6 . At this operation point, the modulation index is set at 0.9. As shown in the figure, the total loss of TCM exceeds that of CCM when electrical angular $\omega t$ increases, and such loss difference reaches its peak when $\omega t$ equals to $0.5 \pi$ due to the increased conduction loss of the TCM strategy.

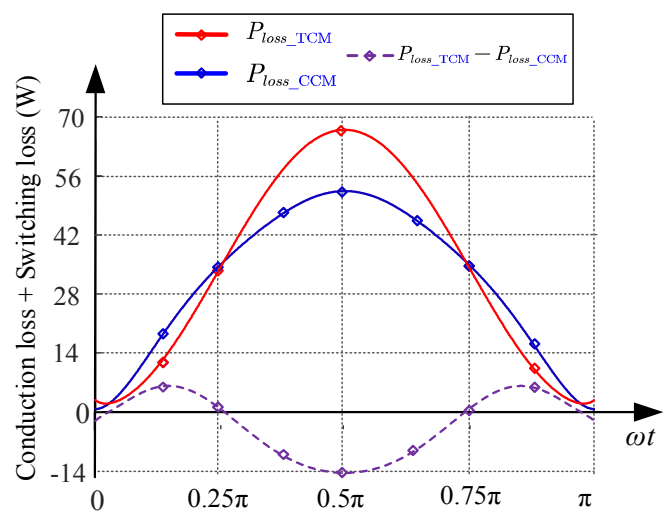

Figure 6. Loss distribution of CCM @ $f_{s w}=15 \mathrm{kHz}$ and TCM over half one line cycle. In this operation condition, the inverter outputs rated current, i.e. 20A.@operation point I (defined in Section III.C) 


\section{Evaluation of the Proposed Strategy from the View of Current Ripple}

According to the discussion in subsection $B$, the instantaneous efficiency is increased by using CCM modulation near the middle of one half cycle, apart from the advantage in reduced current ripple. In practice, the transition between TCM/CCM modulation could be determined by two factors: current ripple and loss distribution in one line cycle.

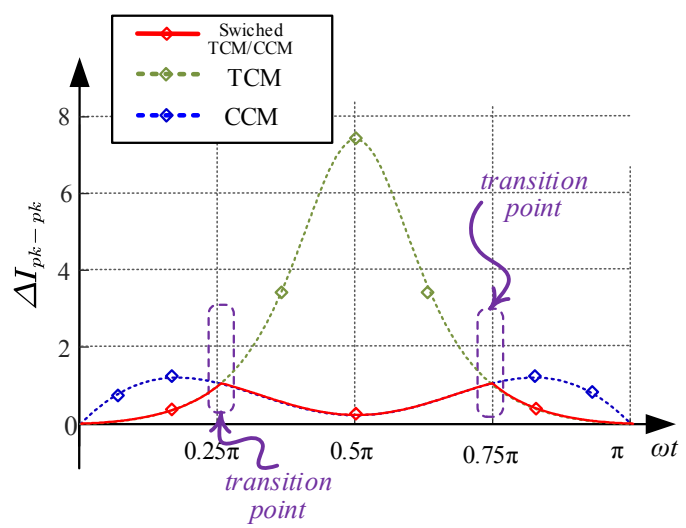

Figure 7. Current ripple distribution of the switched TCM/CCM strategy over half one line cycle.@operation point I

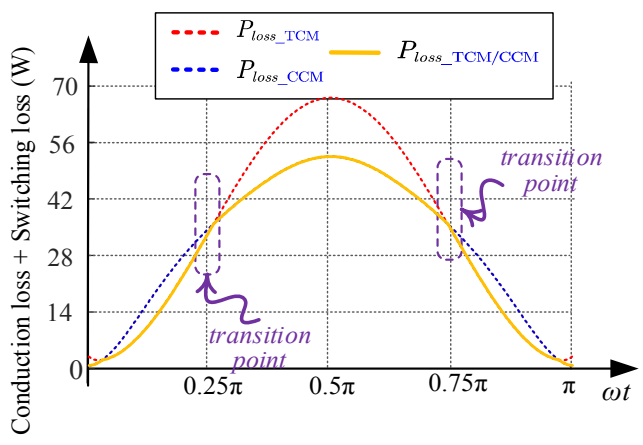

Figure 8. Loss ripple distribution of the proposed switched TCM/CCM strategy over half one line cycle. @ operation point I

In the control design, the maximum allowable current ripple is set at $\Delta I_{p k-p k} / I_{\text {phase } \_ \text {rated }}=7 \%$ and, based on this constraint, the modulation strategy with lower loss is chosen. In this subsection, two switching points are taken as examples.

At operation point I, rated current $I_{\text {rated }}$ is employed and modulation index $m_{a}$ is set to be 0.9 . A comparison of the loss distribution is given in Fig. 6 and a comparison of current ripple distribution is provided in Fig. 7. This operation point corresponds to the high-speed range of the machine and the loss distribution of the switched strategy is shown in Fig. 8. It can be observed that both current THD and inverter efficiency are improved with the proposed switched TCM/CCM strategy.

At operation point II, the output current is kept at $I_{\text {rated }}$ and the modulation index $m_{a}$ is set to be 0.4 , which corresponds to the low-speed operation range of the machine.

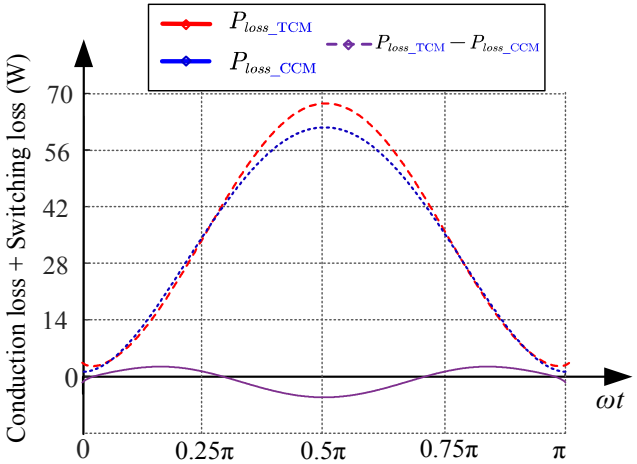

Figure 9. Loss distribution of CCM @ $f_{s w}=15 \mathrm{kHz}$ and TCM over half one line cycle.@operation point II

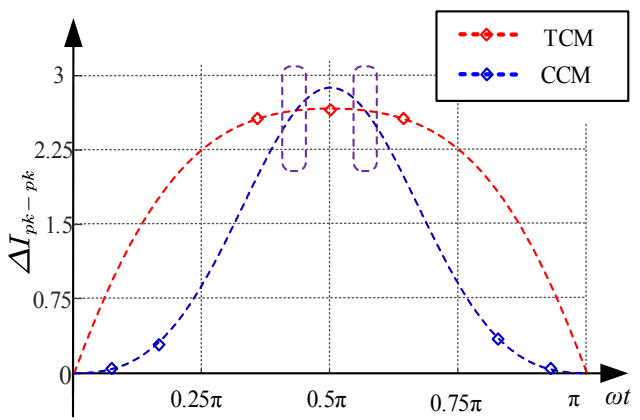

Figure 10. Current ripple distribution of the switched TCM/CCM strategy over half one line cycle. @ operation point II

From Fig. 9 and Fig. 10, two justification of inhibiting switching between the TCM and CCM for low-speed operation point II are observed. Firstly, CCM strategy does not show a considerable superior current ripple performance over TCM strategy over the range where the conduction loss of CCM is smaller than that of TCM. Secondly, the conduction loss of CCM strategy increases as a result of the larger current ripple on the inverter side. This feature indicates that interchanging between CCM and TCM does not yield many gains in terms of current ripple and efficiency when the modulation index is low. The switched modulation strategy is more suitable for a high modulation, i.e. high motor speed, operation point, while the unitary CCM is preferable for the low motor speed operation.

\section{SiMULATION VERIFICATION AND EXPERIMENTAL PLATFORM}

To verify the concept and the proposed modulation strategy quantitatively, simulations are conducted based on PLECS following the specifications given in Table I and II. Simulation results and comparisons are provided in Table III. As shown, the switching strategy can provide superior performance in high modulation operation in terms of efficiency and current experimental ripple. Fig. 11 gives a photo of the constructed setup, based on which experimental results are expected to be provided in the future work. 
TABLE I. SIMULATION RESULTS AND PERFORMANCE COMPARISON @ OPERATION POINT I

\begin{tabular}{ccc}
\hline \hline Modulation Strategy & Current THD & $\begin{array}{c}\text { Inverter } \\
\text { Efficiency }\end{array}$ \\
\hline CCM & $11.5 \%$ & $98.1 \%$ \\
TCM & $9.3 \%$ & $98.4 \%$ \\
Switched TCM/CCM & $4.7 \%$ & $98.7 \%$ \\
\hline \hline
\end{tabular}
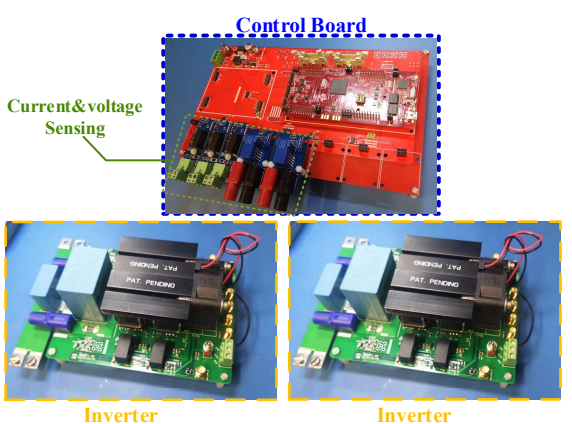

Figure 11. Experimental platform

\section{CONCLUSIONS AND FUTURE WORK}

In this paper, a novel switched TCM/CCM strategy has been proposed. The current ripple and loss distribution in the time domain is investigated analytically under CCM and TCM operations. It is found that the CCM operation shows a low current ripple magnitude in high and low modulation index ranges and a lower overall conduction loss, while the TCM exhibits a greatly lower switching loss and the highest conduction loss difference in the middle of half-line cycle. Based on this feature, a novel switched TCM/CCM strategy is constructed to further enhance the efficiency and decrease the current THD of a traditional TCM inverter for machine drive purposes.

The advantage of the proposed strategy is the reduction of the loss and current THD, which makes the strategy a potential candidate for high-speed machine drive where high fundamental frequency, high efficiency, and low current ripple are desired.

\section{ACKNOWLEDGE}

The paper is under the financial sponsorship of the Jardine Foundation and Cambridge Trust.

\section{REFERENCES}

[1] Y. Jiang and E. L. Severson, "Floating Capacitor Suspension Inverter for Parallel Combined Winding Bearingless Motors," IEEE Transactions on Industry Applications, pp. 1-1, 2019, doi: 10.1109/tia.2019.2957265.

[2] M. Cheng, W. Hua, J. Zhang, and W. Zhao, "Overview of StatorPermanent Magnet Brushless Machines," IEEE Transactions on
Industrial Electronics, vol. 58, no. 11, pp. 5087-5101, 2011, DOI: 10.1109/tie.2011.2123853.

[3] S. Li, Y. Li, W. Choi, and B. Sarlioglu, "High-Speed Electric Machines: Challenges and Design Considerations," IEEE Transactions on Transportation Electrification, vol. 2, no. 1, pp. 2-13, 2016, doi: $10.1109 /$ tte.2016.2523879.

[4] S. Zhu, H. Wang, J. Zhang, Z. Lu, and M. Cheng, "Fast Calculation of Carrier Harmonic Loss in Permanent Magnet of IPMSM Under PWM VSI Supply Over Entire Working Range," IEEE Transactions on Energy Conversion, vol. 34, no. 3, pp. 1581-1592, 2019, doi: 10.1109/tec.2019.2901299.

[5] Y. Yao, F. Peng, and Y. Huang, "Position and Capacitor Voltage Sensorless Control of High-Speed Surface-Mounted PMSM Drive with Output Filter," in 2018 IEEE Energy Conversion Congress and Exposition (ECCE), Portland, OR, USA, 2018.

[6] Z. Zhang, J. Zhang, S. Shao, and J. Zhang, "A High-Efficiency SinglePhase T-Type BCM Microinverter," IEEE Transactions on Power Electronics, vol. 34, no. 1, pp. 984-995, 2019, doi: $10.1109 /$ tpel.2018.2824342.

[7] Z. Huang, Q. Li, and F. Lee, "Critical-Conduction-Mode-Based SoftSwitching Modulation for Three-Phase PV Inverters with Reactive Power Transfer Capability," IEEE Transactions on Power Electronics, pp. 1-1, 2019, doi: 10.1109/tpel.2019.2951130.

[8] C. Marxgut, F. Krismer, D. Bortis, and J. W. Kolar, "Ultraflat Interleaved Triangular Current Mode (TCM) Single-Phase PFC Rectifier," IEEE Transactions on Power Electronics, vol. 29, no. 2, pp. 873-882, 2014, doi: 10.1109/tpel.2013.2258941.

[9] Q. Wang and R. Burgos, "A Method for Increasing Modulation Index of Three Phase Triangular Conduction Mode Converter," in 2018 IEEE 19 th Workshop on Control and Modeling for Power Electronics (COMPEL), Padua, 2018.

[10] M. Kaufmann, A. Tüysüz, and J. W. Kolar, "New optimum modulation of three-phase ZVS triangular current mode GaN inverter ensuring limited switching frequency variation," in 8th IET International Conference on Power Electronics, Machines and Drives (PEMD 2016), Glasgow, 2016.

[11] H. Toyoda, M. Terada, R. Iijima, T. Isobe, and H. Tadano, "Sinusoidal Voltage Output Inverter for Motor Drives Using Discontinuous Current Mode Operation," in 2019 IEEE Applied Power Electronics Conference and Exposition (APEC), Anaheim, CA, USA, 2019.

[12] Z. Huang, Z. Liu, F. C. Lee, and Q. Li, "Critical-Mode-Based SoftSwitching Modulation for High-Frequency Three-Phase Bidirectional AC-DC Converters," IEEE Transactions on Power Electronics, vol. 34, no. 4, pp. 3888-3898, 2019, doi: 10.1109/tpel.2018.2854302.

[13] K. Sabi and D. Costinett, "Design and Implementation of a BipolarUnipolar Switched Boundary Current Mode (BCM) Control GaN-Based Single-Phase Inverter," in 2019 IEEE Energy Conversion Congress and Exposition (ECCE), Baltimore, MD, USA, 2019.

[14] M.-Y. Park, M.-H. Chi, J.-H. Park, H.-G. Kim, T.-W. Chun, and E.-C. Nho, "LCL-filter design for grid-connected PCS using total harmonic distortion and ripple attenuation factor," in The 2010 International Power Electronics Conference - ECCE ASIA -, Sapporo, 2010.

[15] Z. Zhang, M. Chen, M. Gao, Q. Mo, and Z. Qian, "An optimal control method for grid-connected photovoltaic micro-inverter to improve the efficiency at light-load condition," in 2011 IEEE Energy Conversion Congress and Exposition, Phoenix, AZ, 2011.

[16] Y. Shen, L. Shillaber, H. Zhao, Y. Jiang, and T. Long, "Desynchronizing Paralleled GaN HEMTs to Reduce Light-Load Switching Loss," IEEE Transactions on Power Electronics, vol. 35, no. 9, pp. 9151-9170, 2020, doi: 10.1109/tpel.2020.2970240. 